\title{
Influência da interconexão de turbinas eólicas tipos i, ii, iii e iv em parâmetros da capacidade de hospedagem de sistemas de distribuição
}

Adriano A. F. de Moura ${ }^{[1]}$, Herick T. Q. Lemos $^{[2]}$, Antônio M. F. Filho ${ }^{[3]}$, Ailson P. de Moura ${ }^{[4]}$, Victor de P. B. Aguiar ${ }^{[5]}$

${ }^{[1]}$ Universidade Federal Rural do Semi-árido; adrianoaron@ufersa.edu.br

[2] Universidade Federal Rural do Semi-árido; herick.lemos@ufersa.edu.br

[3] Instituto Federal de Educação, Ciência e Tecnologia do Ceará; antonio.filho@ifce.edu.br

${ }^{[4]}$ Universidade Federal do Ceará; ailson@ dee.ufc.br

${ }^{[5]}$ Universidade Federal Rural do Semi-árido; victor@ ufersa.edu.br

Recebido: 12/07/2019;

Aceito: $27 / 07 / 2019$;

Publicado: 07/10/2019.

Resumo: A capacidade de hospedagem de um sistema elétrico de distribuição é o número máximo de geradores distribuídos, que podem ser ligados ao sistema, sem violação de parâmetros operacionais do sistema. Neste trabalho estes parâmetros são: a tensão, o fator de desequilíbrio de tensão e as perdas elétricas. Apesar do estabelecimento desse conceito, não está muito claro na literatura, quais são os impactos, que diferentes tecnologias de turbinas eólicas, podem causar nesses parâmetros da capacidade de hospedagem, sendo este o principal objetivo deste artigo. Para realizar essa avaliação um programa de fluxo de carga trifásico considerando a técnica Backward/Forward foi desenvolvido para o cálculo das variações nos parâmetros. $\mathrm{O}$ sistema de distribuição IEEE 13 barras foi utilizado nos estudos. Uma comparação entre a tecnologia síncrona e de indução em diversas situações é realizada. Os resultados revelam que, em geral, o sistema opera melhor com turbinas conectadas através de conversor de frequência e com regulador na subestação.

Palavras-chave: sistemas de distribuição; capacidade de hospedagem; fluxo de carga

\section{INTRODUÇÃO}

$\mathrm{C}$

OM o advento de novas tecnologias no setor da geração eolioelétrica, impulsionado pelo crescente uso

desta fonte e pela recente competitividade perante as outras fontes de energia, várias linhas de pesquisas surgiram com o objetivo de estudar os impactos da integração de parques eólicos aos sistemas de transmissão e distribuição de energia. Alguns desses trabalhos são listados a seguir.

A aplicação do método da capacidade de hospedagem para um sistema de distribuição realístico, avaliando-se o grau de geração distribuída conectada à rede que pode ser aceito sem causar comprometimento da confiabilidade ou qualidade da energia, considerando-se limites de sobretensão e sobrecorrente para capacidade de hospedagem do sistema [1].

Em [2] é identificado as circunstâncias na qual a capacidade de hospedagem da rede local torna-se restrita à limites de tensão em regime permanente. Além disso, o artigo aborda o controle de tensão de parques eólicos, que pode ser usado para superar tais limitações, aumentando a capacidade de hospedagem local. Para responder a essas perguntas, os dados de um sistema espanhol real são usados em diferentes modelos de rede de crescente complexidade [2].

Em [3] é proposto o uso de estratégias de gestão de ativos (AMSS) para aumentar a capacidade de hospedagem de sistemas de distribuição. Esta referência incorpora AMSS em dois modelos de otimização, cujos objetivos são maximizar o benefício líquido do operador do sistema de distribuição e do proprietário do parque eólico. As estratégias consideradas são: o cerceamento da energia do vento, o controle de tensão coordenada em carga do comutador (on load tap changer) e compensação de energia reativa. 
Apesar das contribuições dos estudos anteriores para o esclarecimento de diferentes aspectos da capacidade de hospedagem, a quantificação dos fenômenos pertinentes à interconexão de turbinas eólicas dos tipos I, II, III e IV ainda não apresenta uma análise mais detalhada na literatura. Neste artigo será realizada a simulação do fluxo de carga de um sistema de distribuição com geração eolioelétrica integrada. O estudo de fluxo de cargas será realizado utilizando-se a técnica iterativa Backward/Forward descrita na referência [4]. O artigo está organizado como segue: A Seção 2 apresenta as características gerais dos modelos de turbinas eólicas empregados nas simulações deste trabalho. A Seção 3 descreve a metodologia utilizada na realização das simulações. A Seção 4 analisa os resultados obtidos nas simulações e, por último, na Seção 5, importantes conclusões são reportadas.

\section{ACOPLAMENTO DE MÁQUINAS DOS TIPOS I, II, III E IV}

As turbinas eólicas são classificadas conforme suas tecnologias construtivas e tipos de geradores empregados, os quais podem ser de indução (GI) ou síncronos (GS). As turbinas eólicas tipo I, II e IV, apresentada na Figura 1, são conectadas diretamente à rede elétrica, ou seja, não se utilizam de conversores de frequência. Tal conexão se caracteriza por ser uma conexão rígida, em que a frequência de operação é fortemente determinada pela rede elétrica. A Seção 2.1 analisará em mais detalhes a conexão dessas arquiteturas à rede elétrica.

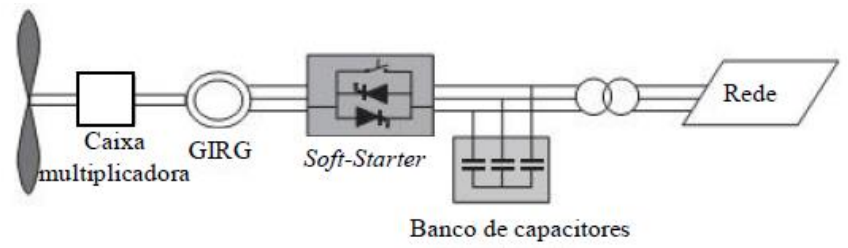

(a)

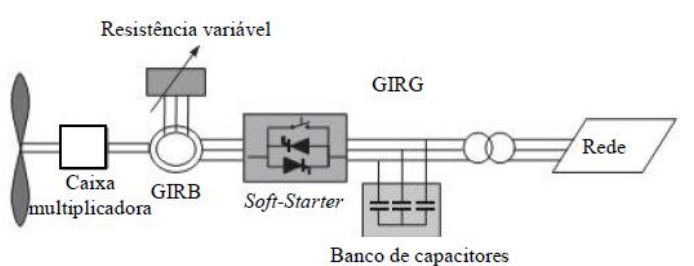

(b)

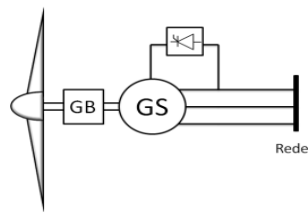

(c)

FIGURA 1. Arquitetura das turbinas eólicas tipos I, II e IV. (a) Gerador de indução com rotor em gaiola (Tipo I) [10]; (b) gerador de indução com rotor bobinado e resistência de rotor controlada (Tipo II) [10]; (c) gerador síncrono diretamente conectado no sistema elétrico (Tipo IV) [1].

As turbinas do tipo III e IV são caracterizadas por possuírem geradores que operam a uma velocidade variável devido a utilização de conversores de frequência. A Figura 2 ilustra os blocos constituintes da arquitetura tipo IV. A turbina tipo III (GIDA) também utiliza conversores de frequência e, muito comumente, um transformador trifásico. A Seção 2.2 ilustra como modelar esses dois tipos de turbina para a análise em um programa de fluxo de carga trifásico.

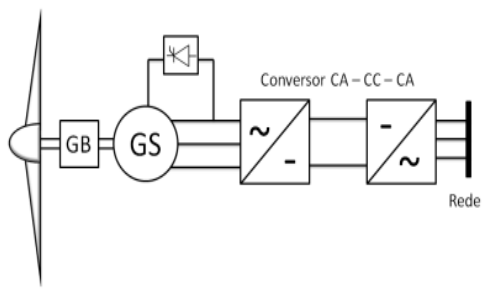

(a)

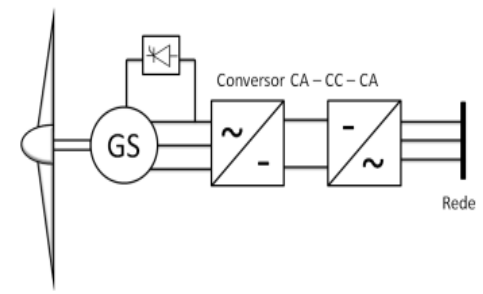

(b)

FIGURA 2. Arquitetura das turbinas eólicas tipo IV. (a) Máquina síncrona conectada via conversor de frequência e caixa de engrenagens (tipo IV) [1]; (b) máquina síncrona conectada via conversor de frequência (tipo IV) [1]. 


\subsection{Conexão direta}

A conexão direta das turbinas se caracteriza como uma conexão rígida com a rede elétrica, pois somente uma reduzida variação na velocidade de rotação do eixo mecânico é permitida. Nesta forma de conexão, grande parte das flutuações do vento é transmitida à rede elétrica.

Para a modelagem dos geradores assíncronos (tipos I e II), a modelagem usada foi adotada [4] (gerador de indução em gaiola de esquilo com/sem resistores externos). A diferença entre os modelos dos tipos I e II é a presença de um resistor externo conectado no circuito do rotor da turbina tipo II. O valor do resistor é ajustado de acordo com determinadas características de operação desejadas.

A modelagem matemática da conexão da máquina síncrona ao sistema elétrico de distribuição do IEEE foi baseada nos trabalhos de Chen [5], cujo modelo tem como propósito demonstrar adequadamente o comportamento da conexão da turbina eólica sem conversor de frequência ao barramento do sistema. Dessa forma, esta seção baseia-se totalmente em seus trabalhos acerca do estudo da geração distribuída em sistemas de distribuição. Todos os componentes do sistema são modelados com seus circuitos equivalentes em termos de indutância, capacitância, resistência e corrente injetada.

$\mathrm{Na}$ literatura, verifica-se em trabalhos acerca do controle de tensão em sistemas com geração distribuída a partir de máquinas síncronas que essas máquinas não são controladas para manter a tensão constante, mas sim a potência e fator de potência constantes. Esses parâmetros são pré-estabelecidos pelas operadoras do sistema. Como resultado, os geradores síncronos podem ser representados aproximadamente como dispositivos de potência constante no estudo do fluxo de carga, ou seja, são representados como dispositivos PQ.

A Figura 3 apresenta o circuito equivalente de Norton de um gerador síncrono modelado com uma fonte de corrente interna, $\mathrm{I}_{\mathrm{I}}^{\mathrm{abc}}$, e uma admitância síncrona, $\mathrm{Y}_{\mathrm{G}}^{\mathrm{abc}}$.

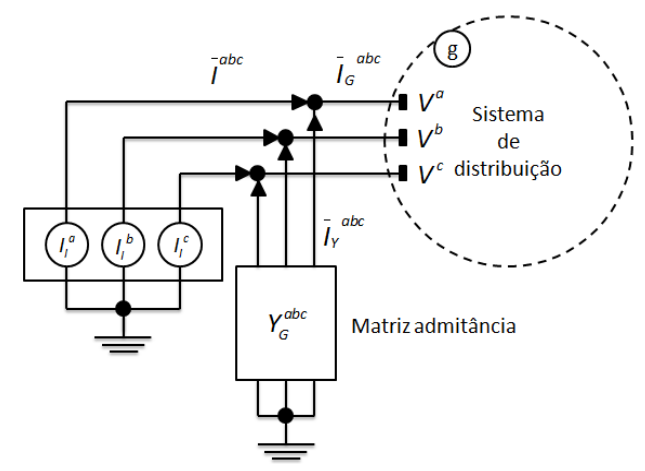

FIGURA 3. Circuito equivalente de Norton de um gerador síncrono [5].

Para modelo da Figura 3, a corrente de linha injetada no sistema de distribuição é calculada por:

$$
\mathrm{I}_{\mathrm{G}}^{\mathrm{abc}}=\frac{\mathrm{P}_{\mathrm{T}-\mathrm{j}} \mathrm{Q}_{\mathrm{T}}}{\frac{\sqrt{3}}{3}\left(\mathrm{~V}_{\mathrm{a}}^{*}+\alpha \mathrm{V}_{\mathrm{b}}^{*}+\alpha^{2} \mathrm{~V}_{\mathrm{c}}^{*}\right)}\left[\begin{array}{c}
1 \\
\alpha^{2} \\
\alpha
\end{array}\right]
$$

Sendo $\alpha$ o operador da transformação de Fortescue, e $\mathrm{P}_{\mathrm{T}}$ e $\mathrm{Q}_{\mathrm{T}}$, respectivamente, as potências ativa e reativa injetadas na rede de distribuição.

A corrente total injetada pela fonte de corrente da Figura 3 é dada por:

$$
\mathrm{I}^{\mathrm{abc}}=\mathrm{I}_{\mathrm{Y}}^{\mathrm{abc}}+\mathrm{I}_{\mathrm{G}}^{\mathrm{abc}}
$$

Com a corrente na admitância shunt, $\mathrm{I}_{\mathrm{Y}}^{\mathrm{abc}}$, dada por:

$$
\mathrm{I}_{\mathrm{Y}}^{\mathrm{abc}}=\mathrm{Y}_{\mathrm{G}}^{\mathrm{abc}} \mathrm{V}_{\mathrm{g}}^{\mathrm{abc}}
$$

Por meio das Equações (1) a (3) pode-se descrever o comportamento das correntes geradas e injetadas no sistema pela máquina síncrona. Maiores detalhes podem ser obtidos na referência [5].

\subsection{Conexão via conversor de frequência}

O conceito da análise do acoplamento da máquina síncrona ao sistema de distribuição via conversor de frequência foi desenvolvido em [6]. Nessa metodologia, que pode ser utilizada pelas turbinas dos tipos III e IV, 
considera-se que a máquina síncrona injeta apenas corrente de sequência positiva, pois para sistemas desequilibrados a quatro fios, o conversor pode determinar as correntes de sequência negativa e zero, atuando de modo a contrabalancear o desequilíbrio causado pelo sistema à jusante do ponto de conexão. Desta forma, as correntes injetadas pela máquina síncrona, por sequência, são dadas por:

$$
\begin{gathered}
\mathrm{I}_{\mathrm{S}^{+}}=\frac{\mathrm{S}^{*}}{3 \mathrm{~V}_{\mathrm{s}^{+}}^{*}} \\
\mathrm{I}_{\mathrm{s} 0, \mathrm{~s}^{-}}=0
\end{gathered}
$$

Sendo $\mathrm{S}$ a potência aparente trifásica injetada pelo conversor, e os subscritos $\mathrm{s}_{0}, \mathrm{~s}_{+}$e $\mathrm{s}$ - utilizados para indicar as componentes de sequência zero, positiva e negativa respectivamente.

Utilizando-se a transformada inversa de Fortescue pode-se facilmente, a partir de (4) e (5), determinar as correntes em componentes de fase injetadas no sistema.

\section{Metodologia}

Para a realização das simulações, o programa ANAREDGEE - Análise de Redes de Distribuição com Geração Eolioelétrica foi escrito e validado de acordo com os resultados do DSACOM do IEEE [7]. O programa implementa todos os modelos necessários para a simulação do sistema IEEE 13 barras e a técnica iterativa Ladder Iterative Technique com o algoritmo de Backward/Forward Sweep para a resolução do fluxo de carga trifásico.

A metodologia utilizada neste trabalho baseia-se, primeiramente, na inserção de turbinas eólicas do tipo IV no sistema modelo IEEE 13 barras da Figura 5. O acoplamento das turbinas eólicas à rede elétrica foi feito a partir de conversores de frequência e de forma direta. Inicialmente, foi simulado a conexão de apenas uma turbina eólica e, posteriormente, simulado a conexão de 2 turbinas eólicas tipo IV.

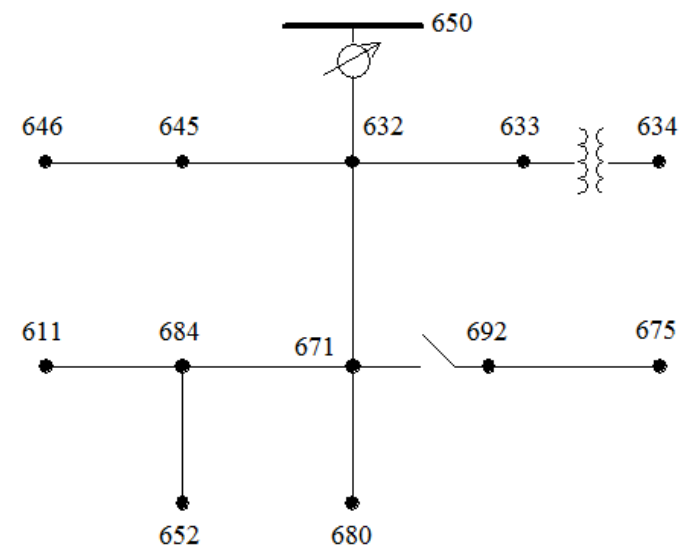

FIGURA 4. Sistema IEEE de 13 barras (Autoria própria).

O sistema modelo trata-se de uma rede de distribuição radial dotada de um regulador de tensão na fonte, de um transformador entre as barras 633 e 634 e de um seccionamento entre as barras 671 e 692 . Todas as características como carregamento das linhas, perfis de tensão em cada barra, etc., estão disponibilizadas em documento fornecido pelo IEEE, denominado IEEE 13 Node Test Feeder, que são considerados como dados de referência para o sistema em sua condição inicial, para que possam ser comparados aos dados resultantes das simulações que contenham a turbina eólica tipo IV. As turbinas foram conectadas na barra 680.

Os resultados obtidos para a turbina eólica tipo III são análogos ao da turbina tipo IV com conversor. Sendo assim, não é necessária nenhuma modelagem adicional, desde que o algoritmo de controle de injeção de corrente de sequência positiva pode ser feito em ambos os tipos de máquinas.

As simulações são realizadas considerando fatores de potência 0.9 capacitivo, 0.9 indutivo e unitário. Os períodos de carga considerados são: pesada (igual ao do sistema original) e leve (a carga é assumida como sendo um terço da carga pesada). 
Em seguida as turbinas dos tipos I e II são simuladas. Os resultados das turbinas do tipo I são comparados diretamente com os resultados da turbina do tipo IV conectada sem conversor de frequência, para que se averigue qual tecnologia provoca maior alteração nos parâmetros analisados relativos à capacidade de hospedagem.

Posteriormente, simulações efetuando-se alterações no próprio sistema de distribuição são realizadas, ou seja: efetua-se o balanço de todas as cargas no alimentador e experimenta-se ligar a máquina de indução e a máquina síncrona com todas as cargas conectadas em delta ou em estrela.

Por último, o efeito da variação do escorregamento, a partir da variação da resistência externa nas máquinas do tipo II, sobre o fator de desequilíbrio da barra 680, é demonstrado. Os dados das máquinas utilizadas neste artigo são apresentados no Apêndice A.

\section{RESULTADOS E DISCUSSÕES}

Foram realizadas 48 simulações do sistema com a variação de diversos parâmetros, como a quantidade de máquinas síncronas conectadas à rede, o fator de potência, presença ou não de regulador de tensão na fonte e carga do sistema. Os valores de referência para o fator de desequilíbrio (conforme a metodologia do Comitê Internacional de Produção e Transmissão de Energia Elétrica - CIGRE), ou seja, quando não há conexão de nenhuma turbina eólica acoplada ao sistema, é de 1,9287\% para o período de carga pesada e de 0,6823\% para o período de carga leve.

As Tabelas 1 e 2 apresentam os resultados referentes ao sistema operando com carga pesada com e sem regulador. Percebe-se que neste caso, o regulador é fundamental para que haja um decréscimo no valor do fator de desequilíbrio tanto no sistema com 1 máquina síncrona quanto no caso em que há 2 máquinas conectadas à barra 680. Todavia, percebeu-se, conforme descrito a seguir, que esta característica não prevalece no sistema quando ele opera em regime de carga leve, principalmente quando a máquina síncrona opera com fator de potência capacitivo.

TABELA 1. Fator de desequilíbrio percentual de tensões para o período de carga pesada com regulador na fonte

(Autoria própria)

\begin{tabular}{ccccc}
\hline \multirow{2}{*}{$\begin{array}{c}\text { Fator de } \\
\text { potência }\end{array}$} & \multicolumn{2}{c}{ 1 máquina síncrona } & \multicolumn{2}{c}{2 máquinas síncronas } \\
\cline { 2 - 5 } & Conexão direta & $\begin{array}{c}\text { Conexão via } \\
\text { conversor }\end{array}$ & Conexão direta & $\begin{array}{c}\text { Conexão via } \\
\text { conversor }\end{array}$ \\
\hline 0,9 indutivo & 1,58 & 1,72 & 1,18 & 1,53 \\
\hline 0,9 capacitivo & 1,66 & 1,80 & 1,45 & 1,67 \\
\hline unitário & 1,61 & 1,56 & 1,26 & 1,57 \\
\hline
\end{tabular}

TABELA 2. Fator de desequilíbrio percentual de tensões para o período de carga pesada sem regulador na fonte (Autoria própria).

\begin{tabular}{ccccc}
\hline \multirow{2}{*}{$\begin{array}{c}\text { Fator de } \\
\text { potência }\end{array}$} & \multicolumn{2}{c}{ 1 máquina síncrona } & \multicolumn{2}{c}{2 máquinas síncronas } \\
\cline { 2 - 5 } & Conexão direta & $\begin{array}{c}\text { Conexão via } \\
\text { conversor }\end{array}$ & Conexão direta & $\begin{array}{c}\text { Conexão via } \\
\text { conversor }\end{array}$ \\
\hline 0,9 indutivo & 2,76 & 2,37 & 3,05 & 2,15 \\
\hline 0,9 capacitivo & 2,77 & 2,38 & 3,12 & 2,19 \\
\hline unitário & 2,78 & 2,35 & 3,13 & 2,11 \\
\hline
\end{tabular}

As Tabelas 3 e 4 apresentam os resultados referentes ao sistema operando com carga leve com e sem regulador. Neste caso, verifica-se que as máquinas conectadas via conversor de frequência tendem a proporcionar um menor fator de desequilíbrio na barra de conexão para ambos os casos com e sem regulador. O pior caso, ou seja, de maior desequilíbrio, ocorre quando há duas máquinas síncronas diretamente conectadas à rede operando com um fator de potência de 0.90 capacitivo e regulador de tensão em operação. Por outro lado, os melhores resultados ocorrem quando o sistema opera com duas máquinas síncronas com um fator de potência de 0.90 indutivo e regulador de tensão fora de operação. 
TABELA 3. Fator de desequilíbrio percentual de tensões para o período de carga leve com regulador na fonte (Autoria própria)

\begin{tabular}{ccccc}
\hline \multirow{2}{*}{$\begin{array}{c}\text { Fator de } \\
\text { potência }\end{array}$} & \multicolumn{2}{c}{ 1 máquina síncrona } & \multicolumn{2}{c}{2 máquinas síncronas } \\
\cline { 2 - 5 } & Conexão direta & $\begin{array}{c}\text { Conexão via } \\
\text { conversor }\end{array}$ & Conexão direta & $\begin{array}{c}\text { Conexão via } \\
\text { conversor }\end{array}$ \\
\hline 0,9 indutivo & 0,58 & 0,51 & 0,49 & 0,36 \\
\hline 0,9 capacitivo & 0,71 & 0,66 & 0,94 & 0,69 \\
\hline unitário & 0,42 & 0,58 & 0,74 & 0,52 \\
\hline
\end{tabular}

TABELA 4. Fator de desequilíbrio percentual de tensões para o período de carga leve sem regulador na fonte (Autoria própria).

\begin{tabular}{ccccc}
\hline \multirow{2}{*}{$\begin{array}{c}\text { Fator de } \\
\text { potência }\end{array}$} & \multicolumn{2}{c}{ 1 máquina síncrona } & \multicolumn{2}{c}{2 máquinas síncronas } \\
\cline { 2 - 5 } & Conexão direta & $\begin{array}{c}\text { Conexão via } \\
\text { conversor }\end{array}$ & Conexão direta & $\begin{array}{c}\text { Conexão via } \\
\text { conversor }\end{array}$ \\
\hline 0,9 indutivo & 0,58 & 0,51 & 0,35 & 0,35 \\
\hline 0,9 capacitivo & 0,76 & 0,66 & 0,93 & 0,69 \\
\hline unitário & 0,66 & 0,58 & 0,70 & 0,52 \\
\hline
\end{tabular}

As perdas ativas e reativas totais do sistema também foram. As menores perdas se concentram na fase B do sistema trifásico, inerentes às próprias cargas conectadas a ela no sistema de distribuição em estudo. Na simulação do sistema em carga pesada, com e sem regulador e com uma ou duas máquinas síncronas eólicas conectadas à rede, verificou-se que, em todas as simulações, as perdas são maiores quando a máquina opera com o fator de potência de 0,9 indutivo.

Por outro lado, quando comparado ao sistema em suas condições iniciais, foi observado que houve uma redução nas perdas totais do sistema em $91 \%$ das simulações realizadas quando trabalhando em carga pesada. O valor original das perdas ativas do sistema é de $110,7 \mathrm{~kW}$ para o período de carga pesada e de $36,049 \mathrm{~kW}$ para o período de carga leve, sendo que, para o período de carga pesada, apenas nas duas simulações mostradas nas Figuras 5 e 6 ocorreram perdas maiores que o valor citado, enquanto que no período de carga leve houve uma redução das perdas totais em todas as situações analisadas.

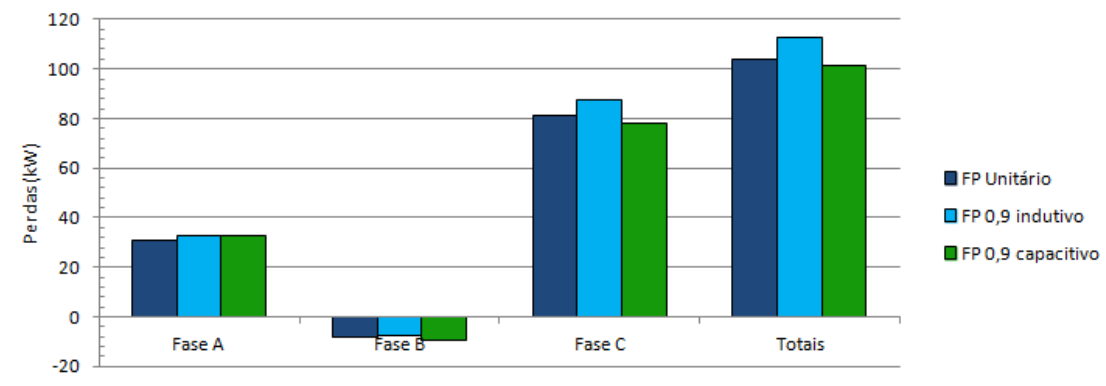

FIGURA 5. Sistema IEEE de 13 barras sem regulador, em horário de carga pesada e com 1 máquinas síncronas diretamente conectadas à rede.

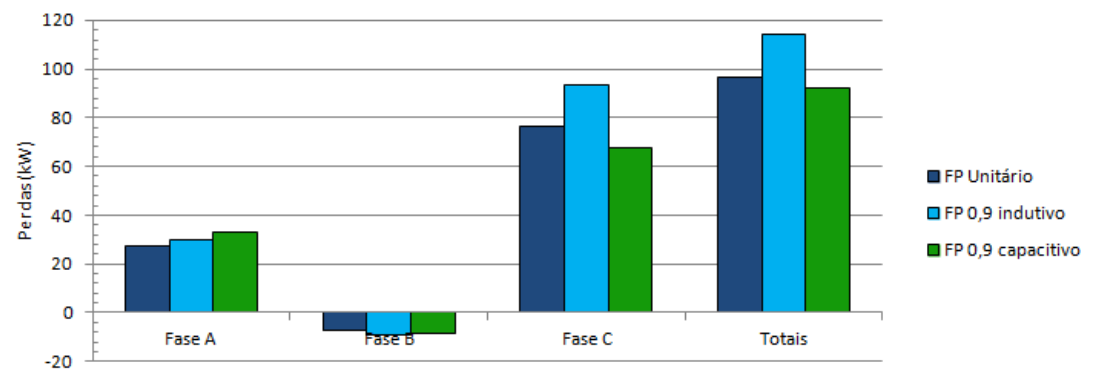

FIGURA 6. Sistema IEEE de 13 barras sem regulador, em horário de carga pesada e com 2 máquinas síncronas diretamente conectadas à rede. 
Outro importante parâmetro analisado foi o perfil das tensões na barra de conexão, no qual foi observado o comportamento tensão com relação à variação do fator de potência da máquina conectada, o tipo de acoplamento, presença ou não de regulador de tensão na fonte e o número de máquinas conectadas na barra 680. Tendo em vista que a Resolução N 505 da ANEEL [8], que dispõe sobre conformidade dos níveis de tensão de energia elétrica em regime permanente, determina como nível adequado de tensão valores compreendidos entre 0,93 e 1,05 em relação ao valor nominal de tensão (1 p.u.), para um nível de tensão superior $1 \mathrm{kV}$ e inferior a $69 \mathrm{kV}$, o valor da tensão na fase B da barra 680 é classificado como crítico para o período de carga pesada, já que o módulo da tensão desta fase é de 1,0529 p.u. nestas condições.

Foi observado que em todas as simulações realizadas em período de carga leve, os perfis das tensões se enquadraram dentro dos valores admissíveis. No entanto, para o período de carga pesada, apenas 3 das 24 situações analisadas propiciaram valores de tensões dentro dos limites. Essas situações tiveram como parâmetros utilizados a presença de 1 e 2 turbinas eólicas com operação em fator de potência de 0,9 capacitivo e de 1 eólica operando em fator de potência unitário, ambos os casos utilizaram a conexão via conversor de frequência e regulador de tensão na fonte. Dentre as combinações dos parâmetros, a situação que mostrou um melhor comportamento das tensões do sistema é apresentada na Figura 7.

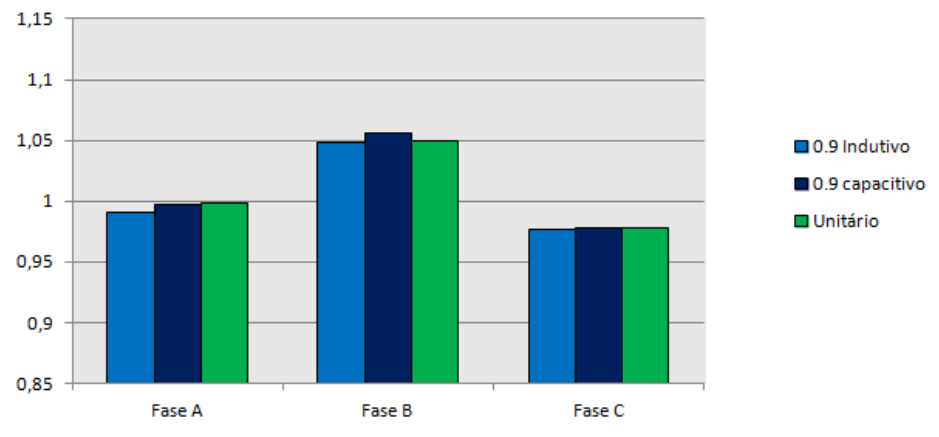

FIGURA 7. Sistema IEEE de 13 barras com regulador, em horário de carga pesada e com 2 máquinas síncronas diretamente conectadas à rede.

Observou-se também que a operação do sistema de distribuição sem regulador de tensão pode ser prejudicial por forçar uma diminuição considerável no valor das tensões, originando valores que se aproximam de 0,885 p.u. na fase A por exemplo, quando o sistema opera com duas turbinas eólicas diretamente conectadas e um fator de 0,90 indutivo. O comportamento das tensões para essa situação é apresentado na Figura 8 . Destaca-se que, independentemente da forma de conexão, a presença de uma turbina eólica no sistema (seja ela dos tipos I, II, III ou IV) contribui para a diminuição do grau de desequilíbrio.

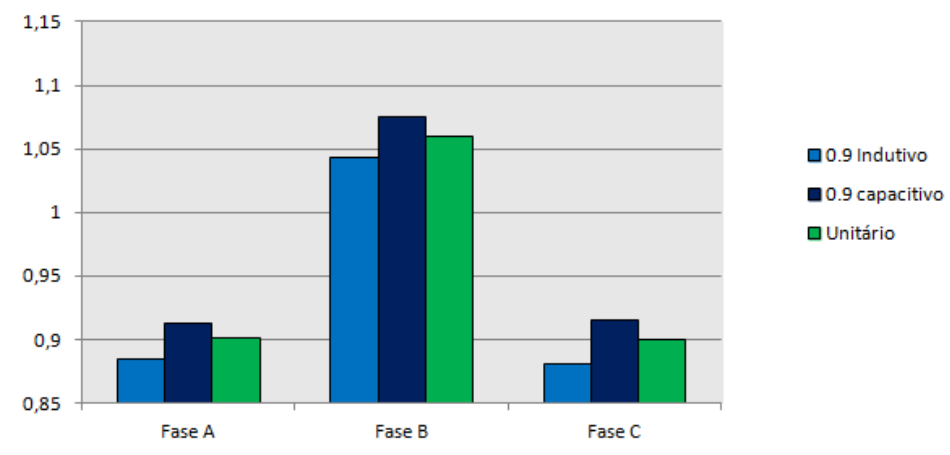

FIGURA 8. Sistema IEEE de 13 barras sem regulador, em horário de carga pesada e com 2 máquinas síncronas diretamente conectadas à rede.

As simulações a seguir visam comparar o desempenho do gerador de indução com o gerador síncrono diretamente conectado. Tomando-se como referência o caso base do sistema IEEE13 barras, foram calculadas as perdas elétricas quando estão conectados o gerador de indução e o gerador síncrono, conforme apresentado nas Tabelas 5 e 6 a seguir. 
Por análise das tabelas, percebe-se que o gerador de indução apresenta menores perdas elétricas tanto de potência ativa quanto de potência reativa, se comparado com o gerador síncrono, quando inserido no sistema. Dentre os casos analisado, a operação da máquina de indução com fator de potência capacitivo apresentou o melhor desempenho. Uma explicação para isto é o fato da potência reativa poder circular nas três fases do sistema na máquina síncrona, aumentando as perdas. Na máquina de indução, por outro lado, ela circula em uma direção e é compensada pela instalação do banco de capacitores.

TABELA 5. Total de perdas para um GI de $500 \mathrm{HP}$ em regime de carga pesada (Autoria própria).

\begin{tabular}{lcccccc}
\hline Fator de & \multicolumn{2}{c}{ Fase A } & \multicolumn{2}{c}{ Fase B } & \multicolumn{2}{c}{ Fase C } \\
\cline { 2 - 7 } potencia & $k W$ & $k V A r$ & $k W$ & $k V A r$ & $k W$ & $k V A r$ \\
\hline 0,98 ind. & 32,548 & 133,661 & $-2,889$ & 32,847 & 66,534 & 109,114 \\
\hline 0,98 cap. & 32,506 & 129,351 & $-3,408$ & 32,680 & 63,813 & 103,478 \\
\hline
\end{tabular}

${ }^{1}$ a - Perdas totais para fator de potência indutivo: 96,194 kW e 275,623 kVAr; b - Perdas totais para fator de potência capacitivo: $92,910 \mathrm{~kW}$ e $265,509 \mathrm{kVAr}$.

TABELA 6. Total de perdas para um GS de 500 HP diretamente conectado à rede em regime de carga pesada (Autoria própria).

\begin{tabular}{l|c|c|c|c|c|c}
\hline \multirow{2}{*}{$\begin{array}{l}\text { Fator de } \\
\text { potência }\end{array}$} & \multicolumn{2}{|c|}{ Fase A } & \multicolumn{2}{c|}{ Fase B } & \multicolumn{2}{c}{ Fase C } \\
\cline { 2 - 7 } & $k W$ & $k V A r$ & $k W$ & $k V A r$ & $k W$ & $k V A r$ \\
\hline 0,98 ind. & 32,548 & 133,661 & $-2,889$ & 32,847 & 66,534 & 109,114 \\
\hline 0,98 cap. & 32,506 & 129,351 & $-3,408$ & 32,680 & 63,813 & 103,478 \\
\hline
\end{tabular}

${ }^{1}$ a - Perdas totais para fator de potência indutivo: 97,864 kW e 281,172 kVAr; b - Perdas totais para fator de potência capacitivo: 95,379 kW e 273,745 kVAr.

A Tabela 7 apresenta um comparativo da influência do tipo de gerado no grau de desequilíbrio na barra 680. Analisando-se essa tabela, nota-se que a inserção do gerador de indução no sistema de distribuição resultou, para o regime de carga pesada, em um menor grau de desequilíbrio se comparado com o gerador síncrono, sendo $1,5570 \%$ para operação com FP igual a 0,98 indutivo e 1,5773\% para o FP igual a 0,98 capacitivo. A Tabela 8 apresenta as perdas elétricas obtidas para máquina de indução.

TABELA 7. Comparativo do grau de desequilíbrio na barra 680 em função do tipo de gerador operando no regime de carga pesada (Autoria própria).

\begin{tabular}{c|c|c}
\hline Fator de potência & $G D(\%)$ do gerador de indução & $G D(\%)$ do gerador síncrono \\
\hline 0,98 ind. & 1,5570 & 1,7817 \\
\hline 0,98 cap. & 1,5773 & 1,8059 \\
\hline
\end{tabular}

TABELA 8. Total de perdas para um GI de 500 HP em regime de carga leve (Autoria própria).

\begin{tabular}{l|c|c|c|c|c|c}
\hline \multirow{2}{*}{$\begin{array}{l}\text { Fator de } \\
\text { potência }\end{array}$} & \multicolumn{2}{|c|}{ Fase A } & \multicolumn{2}{c|}{ Fase B } & \multicolumn{2}{c}{ Fase C } \\
\cline { 2 - 7 } & $k W$ & $k V A r$ & $k W$ & $k V A r$ & $k W$ & $k V A r$ \\
\hline 0,98 ind. & 3,155 & 7,503 & 0,543 & 3,485 & 3,302 & 6,219 \\
\hline 0,98 cap. & 3,289 & 7,029 & 0,674 & 4,258 & 3,290 & 6,865 \\
\hline
\end{tabular}

1 a - Perdas totais para fator de potência indutivo: 7,0 kW e 17,207 kVAr; b - Perdas totais para fator de potência capacitivo: 7,254 kW e 18,152 kVAr.

Examinando-se a Tabela 8, percebe-se que, para o regime de carga leve, as perdas elétricas do sistema caem bruscamente se comparadas com o caso base (cargas pesadas). Já a Tabela 9 apresenta os graus de desequilíbrios do sistema para o regime de carga leve, também na barra 680.

TABEla 9. Comparativo do grau de desequilíbrio na barra 680 em função do tipo de gerador operando no regime de carga leve (Autoria própria).

\begin{tabular}{c|c|c}
\hline Fator de potência & $G D(\%)$ do gerador de indução & $G D(\%)$ do gerador síncrono \\
\hline 0,98 ind. & 0,5653 & 0,6023 \\
\hline 0,98 cap. & 0,6106 & 0,6432 \\
\hline
\end{tabular}


Percebe-se, de acordo com a Tabela 9, que os graus de desequilíbrios para todos os casos foram reduzidos a cerca de um terço dos valores originais. Como exemplo, nota-se uma redução de 66,20\% no grau de desequilíbrio do gerador síncrono operando com fator de potência indutivo.

Com o intuito de estudar o sistema de distribuição operando com carga equilibrada, todas as cargas do sistema (concentradas e distribuídas) do sistema 13 barras foram balanceadas da seguinte forma: em cada nó foi somada as potências ativas (das três fases) e o resultado foi dividido por três e atribuído as respectivas fases A, B e C. O mesmo procedimento foi adotado para as potências reativas presentes em cada nó do sistema. Novamente, foram calculados o fator de desequilíbrio nas barras, as perdas elétricas e os perfis de tensões para o caso de cargas equilibradas.

A Tabela 10 apresenta um resumo dos fatores de desequilíbrio em porcentagem para as várias configurações testadas durante o trabalho, tais como operação do sistema sem regulador de tensão, equilíbrio das cargas nos nós trifásicos e mudança de conexão das cargas (ligação em delta (D) ou estrela (Y)).

TABELA 10. Comparativo do grau de desequilíbrio percentual na barra 680 em função do tipo de gerador e conexão das para o regime de carga pesada (Autoria própria).

\begin{tabular}{|c|c|c|c|c|c|c|c|c|}
\hline \multirow{3}{*}{$\begin{array}{c}\text { Fator } \\
\text { de } \\
\text { potência }\end{array}$} & \multicolumn{4}{|c|}{ Gerador de indução } & \multicolumn{4}{|c|}{ Gerador síncrono } \\
\hline & \multirow{2}{*}{$\begin{array}{l}\text { Operação } \\
\text { sem } \\
\text { regulador }\end{array}$} & \multirow{2}{*}{$\begin{array}{c}\text { Operação } \\
\text { com cargas } \\
\text { equilibradas }\end{array}$} & \multicolumn{2}{|c|}{ Conexão } & \multirow{2}{*}{$\begin{array}{l}\text { Operação } \\
\text { sem } \\
\text { regulador }\end{array}$} & \multirow{2}{*}{$\begin{array}{c}\text { Operação } \\
\text { com cargas } \\
\text { equilibradas }\end{array}$} & \multicolumn{2}{|c|}{ Conexão } \\
\hline & & & $Y$ & $D$ & & & $Y$ & $D$ \\
\hline $\begin{array}{l}0,98 \\
\text { ind. }\end{array}$ & 2,3359 & 1,5614 & 1,4847 & 1,2224 & 2,6945 & 1,7670 & 1,6540 & 1,1439 \\
\hline $\begin{array}{l}0,98 \\
\text { cap. }\end{array}$ & 2,3433 & 1,5432 & 1,4812 & 1,0443 & 2,6972 & 1,7554 & 1,4380 & 1,1547 \\
\hline
\end{tabular}

A Tabela 10 demonstra a importância do regulador para manutenção do nível de desequilíbrio de tensão em valores aceitáveis, com a sua ausência, o grau de desequilíbrio aumentou cerca de $41 \%$ na barra 680 para a operação do sistema com o gerador síncrono. Também se nota a importância do equilíbrio das cargas nos nós do sistema a fim de diminuir o desequilíbrio, pois, no estudo, o equilíbrio das cargas possibilitou uma redução de cerca de $20 \%$ no desequilíbrio de tensão na barra 680 para a simulação com o gerador de indução.

Por fim, também se constatou que uma possível mudança na conexão das cargas do sistema, isto é, em delta ou estrela, também se mostra como uma alternativa eficaz para diminuir o desequilíbrio de tensão no sistema. No estudo, verificou-se que quando todas as cargas foram ligadas em delta, o desequilíbrio decresceu cerca de $46 \%$ para o gerador de indução operando com fator de potência 0,98 capacitivo.

Para avaliação da operação da máquina do tipo II, tendo em vista que não é possível comparar diretamente suas características de operação com os demais geradores, utilizou-se os seguintes dados: resistência externa variando com intervalos de $0,1 \Omega$ partindo de $0,7124 \Omega$ (valor de placa do gerador de $500 \mathrm{hp}$ ) e um escorregamento variando entre $-2 \%$ e $-4 \%$ com intervalos de $-0,5 \%$. A Figura 9 apresenta os resultados obtidos para o grau de desequilíbrio para essa máquina.

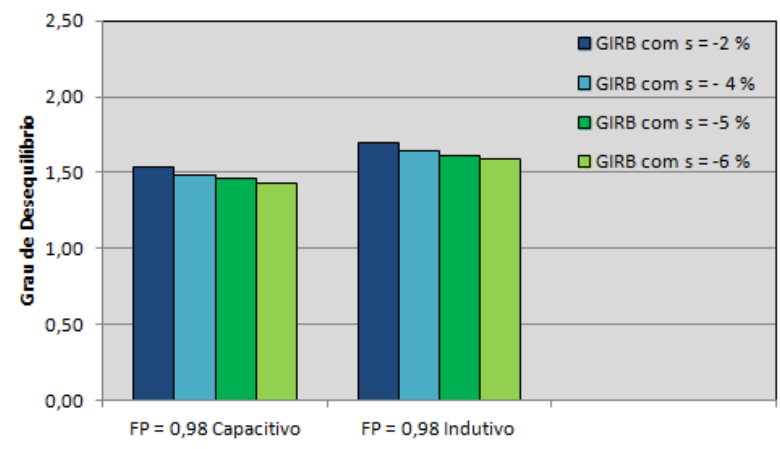

FigURA 9. Sistema IEEE de 13 barras sem regulador, em horário de carga pesada e com 2 máquinas síncronas diretamente conectadas à rede.

Analisando-se a Figura 10, percebe-se que o GIRB, quando inserido na barra 680, diminui o fator de desequilíbrio (do caso base), principalmente para um fator de potência capacitivo. A análise do GIRB pode ser 
realizada para um escorregamento que proporcione a corrente nominal do gerador e pode alcançar $-6 \%$ como em [9]. O escorregamento que proporciona a corrente nominal para o gerador analisado é de $-6 \%$.

\section{CONCLUSÃO}

Através das simulações, verificou-se que o sistema de 13 barras do IEEE sofre maior alteração dos seus parâmetros no regime de carga pesada, principalmente quando há a ausência de regulador de tensão na fonte. Em termos de perdas, percebeu-se que quando as máquinas operam com fator de potência capacitivo, há uma maior redução nas perdas por potência ativa totais do sistema. Para esta situação, também foi verificado que as perdas são reduzidas ainda mais quanto maior for a potência injetada na barra de conexão.

Com relação ao fator de desequilíbrio da barra de conexão, verificou-se que a presença do regulador de tensão é fundamental para que haja uma redução no fator de desequilíbrio, de forma a manter os valores dentro dos limites admissíveis definidos pelos órgãos reguladores. Para um sistema dotado de regulador de tensão, a máquina síncrona diretamente conectada à rede apresentou melhores resultados para quando a rede opera em regime de carga pesada, apresentando valor de 1,179\% quando duas máquinas operam com FP de 0.9 capacitivo, valor bem abaixo dos 1,928\% presente no sistema operante nas condições originais.

Este artigo mostrou que a modelagem de cargas, suas conexões, balanceamento das cargas e tipo de gerador utilizado podem influenciar significantemente no fator de desequilíbrio de um sistema de distribuição de energia elétrica, nas perdas globais e nos perfis de tensões. Mostrou-se que o sistema IEEE 13 barras sofre maiores alterações com a operação com carga pesada, especialmente quando é retirado o regulador de tensão no nó fonte. Demonstrou-se ainda que o gerador de indução contribui de forma mais significativa para a diminuição das perdas (em kW e kVAr) e controle d os fatores de desequilíbrios do sistema, principalmente para o regime de cargas leve. Das diversas configurações testadas a fim de melhorar os parâmetros de funcionamento do arranjo estudado, a variação da forma de conexão de todas as cargas (em estrela ou em delta) se mostrou uma alternativa bastante interessante para mitigar os desequilíbrios de tensão, com destaque para as conexões das cargas em delta, que proporcionaram um redução de 45,55\% (para o gerador de indução com fator de potência capacitivo) no desequilíbrio da barra 680 .

\section{APÊNDICE A}

A Tabela A apresenta os dados das máquinas síncronas utilizadas nas simulações. As impedâncias estão em p.u. na base (monofásica) de 100 MVA.

TABELA A. Impedâncias dos GSs (Autoria própria).

\begin{tabular}{c|c|c|c}
\hline$X_{1}$ & $X_{2}$ & $X_{0}$ & $X_{n}$ \\
\hline 1,0 & 0,141 & 0,05 & 0,05 \\
\hline
\end{tabular}

A Tabela B apresenta os dados das máquinas de indução de $500 \mathrm{HP}$ utilizadas nas simulações. As impedâncias estão em Ohms.

TABELA B. Impedâncias dos GIs (Autoria própria).

\begin{tabular}{c|c|c|c|c}
\hline$R_{s}$ & $X_{s}$ & $X_{m}$ & $R_{r}$ & $X_{r}$ \\
\hline 0,8580 & 3,9495 & 176,9093 & 0,6124 & 3,9495 \\
\hline
\end{tabular}

\section{REFERÊNCIAS}

[1] N. Etherden. M. H. J. Bollen. "Increasing the Hosting Capacity of Distribution Networks by Curtailment of Renewable Energy Resources.” Proceedings of IEEE Trondheim PowerTech, 2011.

[2] E. Sáiz-Márin, E. Lobato, and I. Egido. "Local Hosting Capacity Increase by Means of Wind Farm Voltage Control Provision.” IEEE Transactions on Power Systems, Vol. 29, No. 4, 2014.

[3] S. N. Salih, P. Chen, O. Carlson, L. B. Tjernberg, "Optimizing Wind Power Hosting Capacity of Distribution Systems Using Cost Benefit Analysis.” IEEE Transactions on Power Delivery, Vol. 29, No. 3, 2014.

[4] W. H. Kersting, Distribution System Modeling and Analysis, CRC press, 3nd Edition, New York, USA, 2012. 
[5] T. Chen, M. Chen, T. Inoue, P. Kotas, E. A. Chebli. "Three-phase Cogenerator and Transformer Models for Distribution System Analysis". IEEE Trans. Power Delivery, vol. 6.Oct. 1991.

[6] Z. K. Mohamed, I, Reza. "A Unified Three-Phase Power-Flow Analysis Model For ElectronicallyCoupled Distributed Energy Resources" IEEE Trans. Power Delivery, 2010.

[7] DSACOM - $\quad$ IEEE $\quad$ Distribution $\quad$ Test $\quad$ Feeders. Disponível em:http://ewh.ieee.org/soc/pes/dsacom/testfeeders/index.html

[8] ANEEL - Agência Nacional de Energia Elétrica, Resolução No 505/2001 Disponível em: http://www.aneel.com.br

[9] Brahma, S.M.; Klipsch; Chaudhary, M. ; Ranade, S.J. Some findings about equivalencing windfarms with Type 1 and Type 2 induction generators. North American Power Symposium (NAPS), 2011.

[10] ACKERMANN, Thomas. Wind Power in Power Systems. Sweden: Ed. John Wiley and Sons, 2005. 